\section{PROTECTIVE CARE}

These measures are to be used for the patient's protection:

1. Private room.

2. Strict handwashing before patient care.

3. Care by employees free of infection.

4. Door closed at all times.

5. Stethoscopes and other shared equipment wiped off with alcohol before use

6. Double prepping of all needlestick and finger stick sites.

7. Limited visitors: only two at a time, free of infections please wash hands after entering the room.

Housekeeping:

1. Do not enter if you are sick. Call your supervisor.

2. Wear a yellow, disposable gown.

3. Wash hands and wear gloves.

4. Use freshly mixed cleaning solution, clean cloths and a fresh mop.

5. Wipe all surfaces, including:

Side rails

Telephone

Nurse-call bell

Bedside table

Television

Sink

6. Dispose of solution when you clean the toilet.

7. Mop the floor after removing all trash.

THANK YOU.
Figure.

sealed properly, I should think that there is no way to answer with certainty the question posed by Mr. Birnbaum.

My personal view, unsupported by a study, remains (Infection Control 1983; 4(1):9), that a three-month storage time would be entirely safe-assuming the package is not wet, damaged, or dropped on the floor.

George F. Mallison, MPH, PE Consultant, Environmental and Infection Control Glen Rock, New Jersey

\section{Software for Infection Control Data Gathering \\ To the Editor:}

Is there any software for Apple II Plus or TRS-80 Model 1 for infection control data gathering? I am aware of services that will compile this information using cards.

M.H. Moraleda, MD Chairperson

Infection Control Committee Veterans Administration Medical Center Battle Creek, Michigan
Donald L. Kaiser, DrPH, Associate Professor of Medicine, was invited to respond to Dr. Moraleda's query.

I am not aware of any software specifically for infection control data which will run on microcomputers of the size you mention (TRS-80, Apple II Plus). Our reporting systems require substantially larger machines (DEC PDP 11/24, 11/44, 11/70; DEC VAX 11/730, 11/750, 11/780). However, there are several excellent generalpurpose data base software packages (dBase II is a particularly flexible system) with which someone with moderate computer skills could construct a data system for infection control monitoring. The system would be limited by the available disk storage on the machine being used (though large-capacity hard disks are getting cheaper all the time), and would run relatively slowly (probably not an important problem).

I would advise an interested user to contact a software vendor and investigate, but take along someone who understands computers and your own needs to make sure that the system will suit your purposes.
Donald L. Kaiser, DrPH Associate Professor of Medicine Director, Clinical Computing Laboratory University of Virginia School of Medicine Charlottesville, Virginia

\section{"Reasonableness" in Kidney Transplant Precautions \\ To the Editor:}

We are involved in kidney transplants at our medical center and have really struggled to retain "reasonableness" in precautions with these patients. We have succeeded a bit in just getting the staff down to wearing only masks. What we would prefer is our Protective Care (Figure) which emphasizes handwashing.

I would appreciate your opinion on what is reasonable with transplant recipients.

\section{Sara L. Krantz, RN, BSN Hospital Epidemiologist Pitt County Memorial Hospital Greenville, North Carolina}

Sue Crow, RN, BSN, MSN, Associate Editor of Infection Control, was invited to respond to Ms. Krantz' letter.

Attempting reasonability in patient care procedures is somewhat perplexing in today's hospital. The initial response to any problematic patient care activity is "show me a study that proves. .." If research has been done in this area, data are reviewed, validity determined, and appropriate action taken. Unfortunately, very little research has been done in the infection control area. We cannot erroneously assume that since a particular area has not been studied there is not a problem in that area. The question then is how are decisions made without empirical data? Relying on timeproved principles of nursing may be the answer. Some people prefer to call this theoretical rationale-I call it common sense.

Recognizing this state of the art, let us review your guidelines for a com- 